\title{
Fecal microbiota transplantation
} against intestinal colonization by extended spectrum beta-lactamase producing Enterobacteriaceae: a proof of principle study

\author{
Ramandeep Singh ${ }^{1,3^{*} \dagger}$, Pieter F. de Groot ${ }^{2^{*} \dagger}$, Suzanne E. Geerlings ${ }^{3}$, Caspar J. Hodiamont ${ }^{4}$, Clara Belzer $^{5}$,
} Ineke J. M. ten Berge ${ }^{1}$, Willem M. de Vos ${ }^{5}$, Frederike J. Bemelman ${ }^{1}$ and Max Nieuwdorp ${ }^{2,6,7,8}$

\begin{abstract}
Objective: Infections with multidrug-resistant microorganisms are associated with increased hospitalization, medication costs and mortality. Based on our fecal microbiota transplantation (FMT) experience for Clostridium difficile infection, we treated 15 patients carrying ESBL-producing Enterobacteriaceae (ESBL-EB) with FMT. Seven patients underwent a second FMT after 4 weeks when ESBL-EB remained, amounting to a total number of 22 transplants. The objective was decolonization of ESBL-EB.

Results: Three out of fifteen (20\%) patients were ESBL-negative at 1, 2 and 4 weeks after the first transplant, while six out of 15 (40\%) were negative after the second transplant. Comparison of fecal microbiota at baseline and 4 weeks after FMT revealed restoration of microbial diversity after FMT and a microbial shift towards donor composition. Finally, we suggest several possible factors of response to therapy, such as donor-recipient microbiota match and number of FMTs. Therefore, FMT can be an effective treatment in patients carrying ESBL-EB. Response may be determined by microbiota composition and number of FMT procedures.

Trial registration ISRCTN ISRCTN48328635 Registered 11 October 2017, retrospectively registered
\end{abstract}

Keywords: Multidrug resistance microorganisms, Microbiota, ESBL, Fecal microbiota transplantation

\section{Introduction}

The increased prevalence of antimicrobial-resistant microorganisms is one of the most important medical challenges faced by the worldwide infectious disease community [1]. Antibiotic use is the main driving force behind the development of antimicrobial resistance. Extended-spectrum beta lactamase is a group of enzymes that can hydrolyse penicillin and cephalosporin

\footnotetext{
*Correspondence: r.singh@amc.nl; p.f.degroot@amc.nl

${ }^{\dagger}$ Ramandeep Singh and Pieter F. de Groot contributed equally to this work

${ }^{1}$ Renal Transplant Unit, Division of Nephrology, Academic Medical Centre,

Room A3-273, PO box 22660, 1100 DD Amsterdam, The Netherlands

2 Division of Vascular Medicine, Academic Medical Centre, Room F4-256,

PO box 22660, 1100 DD Amsterdam, The Netherlands

Full list of author information is available at the end of the article
}

group antibiotics, rendering these antibiotics ineffective. The presence of extended spectrum beta lactamase (ESBL) genes in Enterobacteriaceae leads to delayed effective treatment of infections with these multiresistant microorganisms, which is associated with higher mortality, longer hospital admission and higher medical expenditures [2-4]. ESBL producers can be cultured from any human body site, however most E. coli causing urinary tract infections (UTI) derive from the patient's gut microbiota [5]. Since systemic antimicrobial exposure may contribute to further development of antimicrobial resistance, antimicrobial-free ESBL decolonization schemes are preferred. In this regard, fecal microbiota transplantation (FMT), in which the intestinal microbiota of a healthy donor is infused through a gastroduodenal tube may be considered. We and others 
have previously shown that FMT can successfully treat recurrent Clostridium difficile infection (CDI), with an overall cure rate of more than $90 \%[6,7]$. To date, over eight case reports and three case series have been published on successful FMT for MDRO decolonization, varying in treatment method, amount of feces administered, number of administrations, preparation (antibiotics and/or lavage) and targeted pathogen [8,9]. Currently several clinical trials are underway to evaluate effectivity of FMT against a range of MDRO (https://clinicaltr ials.gov; NCT02312986, NCT02543866, NCT02461199, NCT02390622, NCT02472600). Only one is a randomized trial with a 'no treatment' group as control group, while the others are prospective cohort studies with a single treatment group. In this pilot study we aimed to investigate decolonization of ESBL-producing bacteria using FMT.

\section{Main text}

\section{Methods}

\section{Patient and donor selection and FMT procedure}

Out of 155 rectal swab ESBL-positive patients in our hospital between 2012 and 2014, those with a lifeexpectancy of at least 6 months were selected. Patients' clinical data was obtained from their hospital case files. Exclusion criteria were negative rectal swab upon retesting, food allergies and severe immunodeficiency. This resulted in 75 patients that were potentially eligible for FMT. Of these, patients 22 declined to participate, twenty had a negative rectal swab culture upon retesting, eleven could not fulfill the logistic requirements of the study [were not physically or practically able to appear at the study visits (10), or had plans to travel abroad during the study(1)], six were severely immunocompromised (CD4 count below 200 cells/ $\mu$ l) or had a life-expectancy of less than 3 months and one had a food allergy. The remaining fifteen patients agreed to undergo FMT. Of these, five (33\%) were renal transplant recipients using immunosuppressive drugs. Thirteen patients suffered from recurrent urinary tract infections. Other patient characteristics and comorbidity can be found in Table 1 . Of 22 pre-FMT cultures, 19 showed an ESBL-producing E. coli. The other three showed Klebsiella pneumoniae (Table 1).

Fecal microbiota transplantation and screening of donors were performed according to the FMT protocol used as previously published [7] (also refer to the Additional file 1: Methods). No antibiotics were administered before or during FMT. When the patient remained ESBLpositive at 1,2 and 4 weeks after the first FMT, a second treatment was proposed. Two male donors were used for all transplantations: donor 1 was the default donor and donated 16 times. Donor 2 was used when donor 1 was unavailable (twice) or after a failed attempt if he was available (four times) (see Table 1 'patient characteristics'). Written informed consent was obtained from all participants. The study was approved by the local ethics committee and conducted at the Academic Medical Center (Amsterdam), in accordance with the Declaration of Helsinki (updated version 2013). ESBL-EB rectal and urine surveillance cultures were taken at week 1,2 , and

Table 1 Patient characteristics

\begin{tabular}{|c|c|c|c|c|c|c|c|c|c|}
\hline$\#$ & Age & Sex & BMI $\left(\mathrm{kg} / \mathrm{m}^{2}\right)^{\mathrm{a}}$ & Comorbidity & ESBL-Producer & $\begin{array}{l}\text { ESBL-neg. }{ }^{\mathbf{b}} \\
\text { after 1st FMT }\end{array}$ & $\begin{array}{l}\text { ESBL-neg. } \\
\text { after 2nd FMT }\end{array}$ & Donor FMT 1 & Donor FMT 2 \\
\hline 1 & 58 & M & 19 & $\mathrm{ESRD}^{d}, \mathrm{PD}^{\mathrm{e}}, \mathrm{CVD}^{\mathrm{f}}$ & E. colig & Y & - & 1 & - \\
\hline 2 & 47 & M & 27 & Tetraplegia, rUTI ${ }^{h}$ & E. coli & N & - & 1 & - \\
\hline 3 & 65 & M & 25 & Renal Tx $x^{j}, r U T I$ & E. coli & N & N & 1 & 1 \\
\hline 4 & 61 & M & 24 & rUTI & $K \cdot p^{\mathrm{j}}$ & $\mathrm{N}$ & - & 1 & - \\
\hline 5 & 29 & $\mathrm{~F}$ & 35 & rUTI & E. coli & N & Y & 1 & 2 \\
\hline 6 & 56 & $\mathrm{~F}$ & 28 & RUTI & E. coli & N & N & 1 & 2 \\
\hline 7 & 70 & $\mathrm{~F}$ & 28 & Renal Tx, rUTI & K.p. E. coli & N & $\mathrm{N}$ & 1 & 2 \\
\hline 8 & 59 & $\mathrm{~F}$ & 20 & Renal Tx, rUTI, HBVk & E. coli & Y & - & 1 & - \\
\hline 9 & 61 & $\mathrm{~F}$ & 28 & rUTI & E. coli & N & Y & 1 & 1 \\
\hline 10 & 57 & $\mathrm{~F}$ & 26 & ESRD, rUTI & E. coli & N & - & 2 & - \\
\hline 11 & 76 & $\mathrm{~F}$ & 23 & rUTI & E. coli & Y & - & 2 & - \\
\hline 12 & 70 & M & 24 & Renal Tx, rUTI, T2D' & E. coli & $\mathrm{N}$ & - & 1 & - \\
\hline 13 & 59 & $\mathrm{~F}$ & 29 & Renal Tx & K.p & N & $\mathrm{N}$ & 1 & 1 \\
\hline 14 & 58 & $\mathrm{~F}$ & 36 & rUTI & E. coli & N & Y & 1 & 2 \\
\hline 15 & 21 & $\mathrm{~F}$ & 24 & rUTI & E. coli & $\mathrm{N}$ & - & 1 & - \\
\hline
\end{tabular}

${ }^{a}$ Body mass index, ${ }^{b}$ extended-spectrum beta lactamase producer-negative, ${ }^{c}$ fecal microbiota transplantation, ${ }^{d}$ end-stage renal disease, ${ }^{e}$ peritoneal dialysis, ${ }^{f}$ cardiovascular disease, ${ }^{\mathrm{g}}$ Escherichia coli, ${ }^{\mathrm{h}}$ recurring urinary tract infections, ${ }^{\mathrm{i}}$ transplant, ${ }^{\mathrm{j}} \mathrm{Klebsiella} \mathrm{pneumoniae}^{\mathrm{k}}{ }^{\mathrm{H}} \mathrm{Hepatitis} \mathrm{B}$ virus, ${ }^{\mathrm{l}}$ type 2 diabetes. Patient 7 had (only) K. pneumoniae before the 1st FMT and (only) E. coli before the second FMT 
4 after FMT. ESBL-EB surveillance culture procedures, FMT procedures and donor selection are discussed in the Additional file 1: Methods.

\section{Microbiota analyses}

Fecal samples were taken at baseline and 4 weeks after FMT. Microbiota were analyzed from these samples in Wageningen University by human intestinal tract (HIT-) chip, a custom-made microarray containing roughly 5500 specific oligonucleotide probes that cover over 1000 intestinal phylotypes, which is used for the high-throughput profiling of the fecal microbiota [10]. For a more detailed description, please refer to the Additional file 1: Methods.

\section{Statistical analyses}

For the microbiota plots we have performed a principal component analyses, which is a commonly used statistical procedure used for graphical representation of microbiota compositional differences between samples, usually depicted as distances between dots in a two-dimensional space showing only principal component 1 and 2 (on the $\mathrm{x}$ - and $\mathrm{y}$-axis), which by definition explain most of the variance. This was done in R-studio (version 0.99.903). Comparison between microbiota at baseline and 4 weeks after FMT was done using Wilcoxon's signed rank test for each HITchip phylotype. P values were corrected for multiple testing using 'qvalue' package in R. Comparison between responder and nonresponder microbiota at baseline and 4 weeks after FMT were done with Wilcoxon's signed rank test.

\section{Results}

\section{Fecal microbiota transplantation}

In total fifteen patients underwent an FMT procedure, of whom seven underwent a second FMT. After the first FMT procedure, decolonization was successful in three patients $(20 \%)$ at all timepoints $(1,2$ and 4 weeks followup), except for subject 1 who showed a delayed response and became negative at week 2 (Additional file 2: Table S4). Patients who remained ESBL-EB negative on follow-up were named 'responders'. The twelve nonresponders remained ESBL-EB positive on all timepoints. Seven out of twelve non-responders underwent a second FMT, three of which led to decolonization at all timepoints (1, 2 and 4 weeks) after the second FMT, resulting in an overall eradication success rate of $40 \%$ Additional file 2: Table S4 and Fig. 1a). Patient characteristics are summarized in Table 1. Subsequent analyses showed that ESBL eradication was associated with having had two FMTs (3/7 or $43 \%$ success rate) instead of one FMT $(3 / 15$ or $20 \%$ success rate) as well as FMT donor specific treatment effects (donor 2 had a $50 \%$ success rate $(3$ out of 6) versus $19 \%$ of donor 1 (3 out of 16)). Moreover, immunocompromised state (e.g. patients with a kidney transplant) was associated with a lower success rate $(1 / 5$ or $20 \%)$. It should be stressed that these findings are not statistically significant and can thus be merely hypothesis-raising. Patient characteristics of responders and nonresponders are reported in Additional file 3: Table S1, success rate and patient characteristics per transplantation are shown in Additional file 4: Table S2. Finally and importantly, no side effects besides mild discomfort and temporary loose stools $(<24 \mathrm{~h})$ were reported after FMT.

\section{Fecal microbiota analysis}

At 4 weeks after FMT, a general shift in fecal microbiota composition towards donor microbial composition was seen in the patients, depicted in Fig. $1 \mathrm{~b}$ as a shift to the left along principal component 1 (PC1) and downward along principal component 2 (PC2). Overall, fecal microbial diversity (Shannon's diversity index) also changed significantly after FMT (Fig. 1c).

Although fecal microbiota composition in responders did not form a separate cluster from the non-responder microbiota in the principal component analysis (Fig. 1d), responder microbiota clustered more tightly (Fig. 1d, inside the purple square). Interestingly, responder microbiota composition resembled donor 2 composition more than donor 1 composition (Fig. 1d) which coincides with the observation that donor 2 seemed the more effective of the two.

In total, 664 bacterial taxa changed significantly after FMT (Wilcoxon's, adjusted q-value $<0.05$ ). Between responders and non-responders 21 taxa were significantly different (Wilcoxon's, $p$ value $<0.05$ ) at 4 weeks after FMT, seven of which had also changed significantly and more than twofold in abundance after FMT (Additional file 5: Figure S1). At baseline, six species were significantly different between responders and nonresponders (Additional file 6: Table S3).

\section{Discussion}

Decolonization of ESBL-EB with antibiotic regiments may be achieved, but is often followed by recolonization [11]. In contrast, FMT may decolonize while preventing relapse and work in synergy with antibiotic regimens, as it does in the treatment of recurrent CDI [12]. In our small explorative study, fecal microbiota transplantation was explored as a novel treatment against colonization by multi-drug-resistant microorganisms (MDRO).

As mentioned in the introduction, several studies have been published on the subject. Noteworthy is a recent case series in which $60 \%$ of 25 patients with blood disorders were decolonized from antibiotic-resistant pathogens when sampled 1 month after FMT [13]. While this 

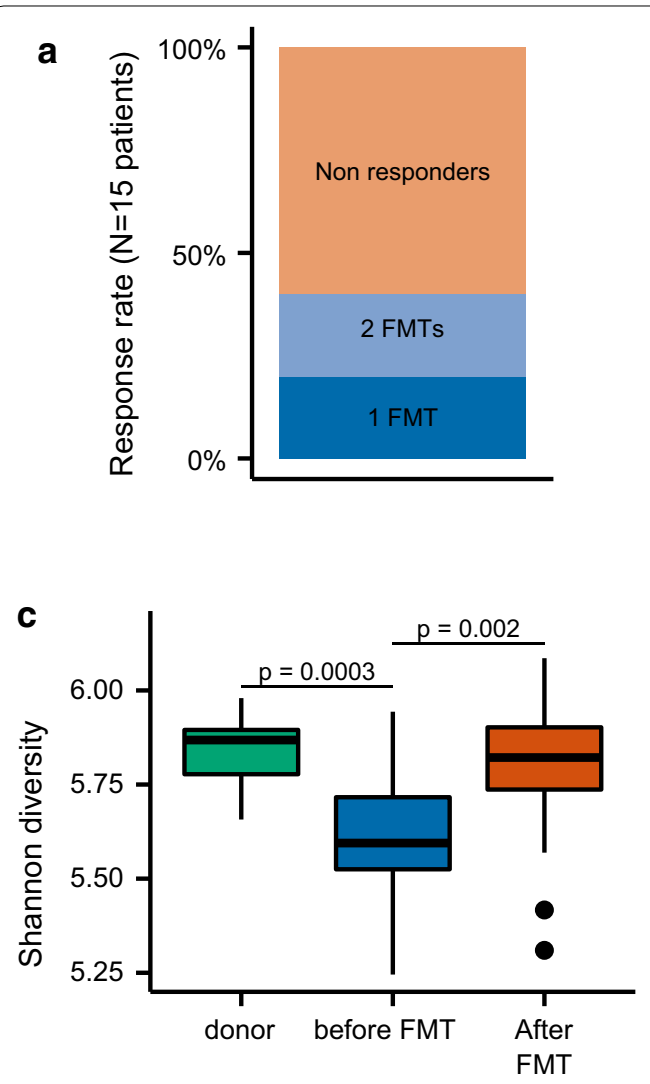

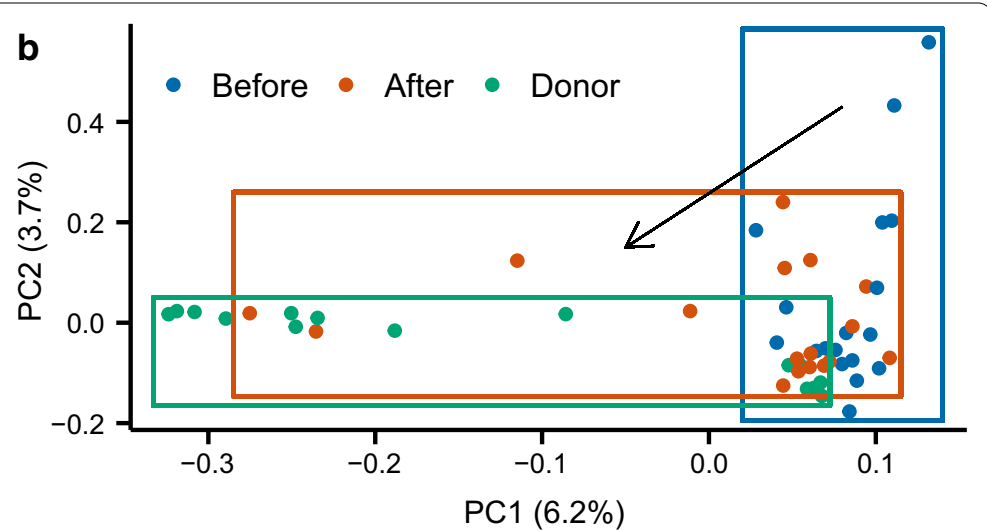

d

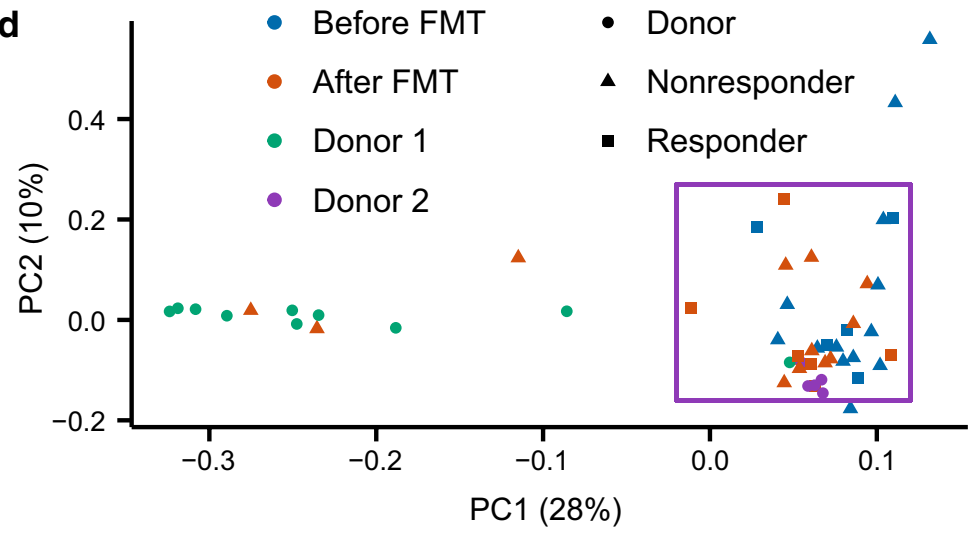

Fig. 1 a Percentage of responders after one and two FMTs. b PCA-plot showing microbiota composition of donors and recipients before and after FMT. c Microbial diversity $\mathbf{d}$ Microbiota of responders and non-responders. The black square encloses all responders

study features a larger sample size, our study sampled earlier after FMT and at several time points, minimizing the chance of spontaneous clearance as explanation for success. Furthermore, none of our subjects used antibiotics concurrently with FMT treatment.

FMT is already successfully being applied for Clostridium difficile infection (CDI), for which it is highly effective [14]. For other indications response rates seem modest thus far. Therefore, factors that predict response or non-response need to be identified, requiring a more detailed analytic approach. In the prospect of larger trials, our small uncontrolled study shows several interesting clues.

Interestingly, a recent case series has shown that five out of five patients cleared MRSA after nasojejunal administration of FMT using three consecutive FMTs [15]. Although it should be noted that in this study pretreatment antibiotics (vancomycin) was used and pathogen type was different, its findings are in line with our finding that repeat-FMT leads to a higher success rate. It should be noted however, that optimal dose, frequency and route of administration of FMT in ESBL is still unclear.

Our study shows that FMT in ESBL-EB colonization restores bacterial diversity, similar to FMT in CDI [7]. To further dissect driving mechanisms, we studied changes in fecal microbial signatures associated with response to FMT and observed that microbiota in responders (as opposed to microbiota in non-responders) cluster in a relatively tight formation and more closely resemble the microbiota of donor 2 (Fig. 1d). We therefore hypothesize that improved donor-recipient match improves FMT success rate and may explain the higher success rate of donor 2 in our study (3/6 vs. $3 / 16$ of donor 1$)$. Finally, seven bacterial taxa had at the same time changed significantly after FMT and were significantly different between responders and non-responders (Additional file 5: Figure S1), including several known butyrate producers.

Thus far, FMT studies generally focus on bacterial composition. It is often postulated, for CDI but also for ESBL, that healthy donor bacteria simply restore diversity and cure the disease by outcompeting pathogens. However, although microbiota composition changed markedly and 
diversity increased after FMT in our study, no distinction between responders and non-responders could be made based on these factors. Furthermore, a recent uncontrolled study convincingly disqualifies direct transfer of bacteria as the curative mechanism in CDI by showing sterile fecal filtrates (obtained by filtration of a preprocessed fecal solution through a $0.2 \mu \mathrm{m}$ filter) derived from donor feces cure CDI just as well (five out of five patients) [16]. This emphasizes the importance of other fecal solutes, such as bacterial products, bacteriophages or other immune system-provoking agents in explaining how FMT can lead to disease resolution.

\section{Conclusion}

In conclusion we have shown an efficacy of (repeat-) FMT in ESBL decolonization of 6/15 (40\%). Our results suggest repeat FMT increases treatment effectivity. Possibly, donor choice, patient characteristics and donor-patient match also play a role. Our study shows microbial diversity restoration after FMT, without relation to response rate. Several microbes are associated with response. In the future, larger randomized-controlled studies should confirm these findings. These analyses should ideally not be limited to characterization of gut bacteria, but also include other active agents in the fecal solution.

\section{Limitations}

Our study has some important limitations. The first limitation is that it lacks a control group. It can be postulated that not FMT but rather spontaneous clearance has caused decolonization in our 'responders'. Indeed, spontaneous clearance of MDRO in previously hospitalized patients after discharge is common (about $20-30 \%$ of cases) $[17,18]$. However, our population consisted of outpatients with prolonged colonization (months to years) in a home setting. Since there was only 1 week between pre-FMT sampling and post-FMT sampling in which this spontaneous clearance could have happened, we dare say that we expect the contribution of spontaneous clearance on our treatment success rate is modest. Secondly, another limitation of our study is the small study group, therefore the factors of success that we identified were not statistically significant. Thirdly, longer follow-up than 4 weeks would provide valuable information on whether FMT protects from relapse after new antibiotic courses. And finally, our population consisted of patients with various and often severe medical conditions, among which several renal transplant subjects. Therefore it is doubtful whether our data can be extrapolated to the general population. That being said, our population is representative in the sense that it is a patient group suffering from recurrent urinary tract infections with ESBL-EB.

\section{Additional files}

Additional file 1: Methods. Additional materials and methods.

Additional file 2: Table S4. Culture results in decolonized subjects. Rectal and urine cultures at follow-up (baseline, 1, 2 and 4 weeks after FMT).

Additional file 3: Table S1. Characteristics of responders vs nonresponders. Characteristics of responders vs nonresponders.

Additional file 4: Table S2. Characteristics of successful FMTs vs unsuccessful FMTs. Characteristics of successful FMTs vs unsuccessful FMTs.

Additional file 5: Figure S1. Heat map of change in responders vs nonresponders. Heatmap showing fold change in microbiota abundance of significantly different species between responders and non-responders before versus after FMT. FMTs are shown, therefore patients who have received 2 FMTs are shown twice. Faecalibacterium 1 and 2 are both subgroups of the Faecalibacterium genus. Faecalibacterium 1 is the genus in the strict sense, whereas the Faecalibacterium 2 group includes uncultured bacteria related to the phylotypes Eldhufec289, Eldhufec276 and Eldhufec259.

Additional file 6: Table S3. Abundances of taxa in responders vs nonresponders. Relative abundances of significantly different taxa (only $<0.9$ or $>1.1$ fold shown) between responders and nonresponders at baseline. Median relative abundance (percentage) is shown, also the ratio between the medians of responders and nonresponders and the $p$-value are given.

\section{Abbreviations}

CDI: Clostridium difficile infection; ESBL: extended spectrum beta lactamase; EB: Enterobacteriaceae; ESBL-EB: extended spectrum beta lactamase producing enterobacteriaceae; FMT: fecal microbiota transplantation; HIT-chip: human intestinal tract chip; HIV: human immunodeficiency virus; MALDI-TOF: matrix assisted laser desorption/ionisation time-of-flight analyzer; MIC: minimum inhibitory concentration; MDRO: multi-drug-resistant microorganisms; MS: mass spectrometer; PC: principal component; UTI: urinary tract infections.

\section{Authors' contributions}

$\mathrm{RS}$ and PFdG performed the fecal transplantations and are responsible for the data collection. RS analyzed and reported most of the clinical data and PFdG performed and reported the microbiota analyses. They jointly wrote most of the manuscript. MN, FJB, SEG, IJMtB conceived the study design, supervised the project and revised the manuscript. WMdV advised on and verified the microbiota analyses and revised the manuscript. CJH provided microbiology advice and wrote part of the methods (on ESBL-EB culturing) and revised the manuscript. CB supervised the HIT-chip analysis and revised the manuscript. All authors read and approved the final manuscript.

\section{Author details}

${ }^{1}$ Renal Transplant Unit, Division of Nephrology, Academic Medical Centre, Room A3-273, PO box 22660, 1100 DD Amsterdam, The Netherlands. ${ }^{2}$ Division of Vascular Medicine, Academic Medical Centre, Room F4-256, PO box 22660, 1100 DD Amsterdam, The Netherlands. ${ }^{3}$ Division of Infectious Diseases, Center of Infection and Immunity Amsterdam (CINIMA), Academic Medical Centre, Amsterdam, The Netherlands. ${ }^{4}$ Department of Medical Microbiology, Academic Medical Centre, Amsterdam, The Netherlands. ${ }^{5}$ Department of Microbiology, Wageningen University, Wageningen, The Netherlands. ${ }^{6}$ Department of Internal Medicine, VU University Medical Center, Amsterdam, The Netherlands. ${ }^{7}$ ICaR-VU, VU University Medical Center, Amsterdam, The Netherlands. ${ }^{8}$ Wallenberg Laboratory, Sahlgrenska Hospital, University of Gothenburg, Gothenburg, Sweden.

\section{Acknowledgements}

We want to acknowledge Guido Bakker for his contributions and Jorn Hartman for doing the HITchip work. 


\section{Competing interests}

WMdV is on the Scientific Advisory Board of Caelus Health. MN is on the Scientific Advisory Board of Caelus Health. All other authors declare that they have no competing interests.

\section{Availability of data and materials}

The datasets used and/or analyzed during the current study are available from the corresponding author on reasonable request.

\section{Consent for publication}

Not applicable.

\section{Ethics approval and consent to participate}

Written informed consent was obtained from all participants. The study was approved by the local ethics committee named "Medisch Ethische Toetsingscommissie Academic Medical Center (METC AMC)" (protocol number: MEC 2013_013\#B2013281) and conducted at the Academic Medical Center (Amsterdam), in accordance with the Declaration of Helsinki (updated version 2013) 017.

This trial was registered at ISRCTN (ISRCTN48328635) on 11th October

\section{Funding}

This work was supported by a Grant from de Nierstichting (Dutch Kidney Foundation), M. Nieuwdorp is supported by a ZONMW-VIDI Grant 2013 (016.146.327) of the Netherlands Organization for Scientific Research (NWO) and CVON Young Talent Grant 2012, WM de Vos is supported by the NWO Gravitation Grant (SIAM 024.002.002) and the Spinoza Award.

\section{Publisher's Note}

Springer Nature remains neutral with regard to jurisdictional claims in published maps and institutional affiliations.

Received: 28 November 2017 Accepted: 13 March 2018

Published online: 22 March 2018

\section{References}

1. Fauci S, Morens MD. The perpetual challenge of infectious diseases. N Engl J Med. 2012;366:454-61.

2. Lautenbach E, Patel JB, Bilker WB, Edelstein PH, Fishman NO. Extendedspectrum $\beta$-lactamase-producing Escherichia coli and Klebsiella pneumoniae: risk factors for infection and impact of resistance on outcomes. Clin Infect Dis. 2001:32:1162-71.

3. Rottier WC, Ammerlaan HSM, Bonten MJM. Effects of confounders and intermediates on the association of bacteraemia caused by extendedspectrum $\beta$-lactamase-producing Enterobacteriaceae and patient outcome: a meta-analysis. J Antimicrob Chemother. 2012;67:1311-20.

4. Paterson DL, Bonomo RA. Extended-spectrum $\beta$-lactamases: a clinical update. Clin Microbiol Rev. 2005;18:657-86.

5. Niki M, Hirai I, Yoshinaga A, Ulzii-Orshikh L, Nakata A, Yamamoto A, et al. Extended-spectrum $\beta$-lactamase-producing Escherichia coli strains in the feces of carriers contribute substantially to urinary tract infections in these patients. Infection. 2011;39:467.

6. Smits LP, Bouter KEC, de Vos WM, Borody TJ, Nieuwdorp M. Therapeutic potential of fecal microbiota transplantation. Gastroenterology. 2013:145:946-53.

7. van Nood E, Vrieze A, Nieuwdorp M, Fuentes S, Zoetendal EG, de Vos WM, et al. Duodenal infusion of donor feces for recurrent Clostridium difficile. $N$ Engl J Med. 2013;368:407-15.

8. Manges AR, Steiner TS, Wright AJ. Fecal microbiota transplantation for the intestinal decolonization of extensively antimicrobial-resistant opportunistic pathogens: a review. Infect Dis. 2016;48:587-92.

9. Stalenhoef JE, Terveer EM, Knetsch CW, Van't Hof PJ, Vlasveld IN, Keller JJ, et al. Fecal microbiota transfer for multidrug-resistant gram-negatives: a clinical success combined with microbiological failure. Open Forum Infect Dis. 2017;4(2):ofx047.

10. Rajilić-Stojanović M, Heilig HGHJ, Molenaar D, Kajander K, Surakka A, Smidt $H$, et al. Development and application of the human intestinal tract chip, a phylogenetic microarray: analysis of universally conserved phylotypes in the abundant microbiota of young and elderly adults. Environ Microbiol. 2009:11:1736-51.

11. Rieg S, Kupper MF, de With K, Serr A, Bohnert JA, Kern WV. Intestinal decolonization of Enterobacteriaceae producing extended-spectrum beta-lactamases (ESBL): a retrospective observational study in patients at risk for infection and a brief review of the literature. BMC Infect Dis. 2015:15:475.

12. van Beurden $\mathrm{YH}$, de Groot PF, van Nood E, Nieuwdorp M, Keller JJ, Goorhuis A. Complications, effectiveness, and long term follow-up of fecal microbiota transfer by nasoduodenal tube for treatment of recurrent Clostridium difficile infection. United Eur Gastroenterol J. 2016;5:868-79.

13. Bilinski J, Grzesiowski P, Sorensen N, Madry K, Muszynski J, Robak K, et al. Fecal microbiota transplantation in patients with blood disorders inhibits gut colonization with antibiotic-resistant bacteria: results of a prospective, single-center study. Clin Infect Dis. 2017;65:364-70.

14. Vrieze A, Van Nood E, Holleman F, Salojärvi J, Kootte RS, Bartelsman JFWM, et al. Transfer of intestinal microbiota from lean donors increases insulin sensitivity in individuals with metabolic syndrome. Gastroenterology. 2012:143(913-6):e7.

15. Wei Y, Gong J, Zhu W, Guo D, Gu L, Li N, et al. Fecal microbiota transplantation restores dysbiosis in patients with methicillin resistant Staphylococcus aureus enterocolitis. BMC Infect Dis. 2015;15:265.

16. Ott SJ, Waetzig GH, Rehman A, Moltzau-Anderson J, Bharti R, Grasis JA, et al. Efficacy of sterile fecal filtrate transfer for treating patients with Clostridium difficile infection. Gastroenterology. 2017;152(4):799-811.e7.

17. Lubbert C, Lippmann N, Busch T, Kaisers UX, Ducomble T, Eckmanns T, et al. Long-term carriage of Klebsiella pneumoniae carbapenemase2-producing $\mathrm{K}$ pneumoniae after a large single-center outbreak in Germany. Am J Infect Control. 2014;42:376-80.

18. Bar-Yoseph H, Hussein K, Braun E, Paul M. Natural history and decolonization strategies for ESBL/carbapenem-resistant Enterobacteriaceae carriage: systematic review and meta-analysis. J Antimicrob Chemother. 2016;71:2729-39

\section{Submit your next manuscript to BioMed Central} and we will help you at every step:

- We accept pre-submission inquiries

- Our selector tool helps you to find the most relevant journal

- We provide round the clock customer support

- Convenient online submission

- Thorough peer review

- Inclusion in PubMed and all major indexing services

- Maximum visibility for your research

Submit your manuscript at www.biomedcentral com/submit
Ciomed Central 